\title{
Correction to: Reversible denoising and lifting based color componenttransformation for lossless image compression
}

\section{Roman Starosolski ${ }^{1}$}

Published online: 13 January 2020

C) Springer Science+Business Media, LLC, part of Springer Nature 2020

\section{Correction to: Multimedia Tools Applications (2019) https://doi.org/10.1007/s11042-019-08371-w}

The phrase above equation 5 in the original article stating "by applying denoising to the arguments of function:" should be corrected as "by applying denoising to the arguments of function f:." The original article has been corrected.

The online version of the original article can be found at https://doi.org/10.1007/s11042-019-08371-w

Roman Starosolski

rstarosolski@polsl.pl; rstaros@ gmail.com

1 Institute of Informatics, Silesian University of Technology, Akademicka 16, 44-100 Gliwice, Poland 